\title{
Trauma Central
}

\author{
Varun S. Mehta ${ }^{1}$
}

Received: 3 October 2021 / Accepted: 30 October 2021 / Publishd online: 9 November 2021

(c) Academic Psychiatry 2021

The blood from my head obscured my name on my medical student identification card, spattering down onto my backpack and clothes.

I was two minutes into my commute to the hospital that bright summer morning, scootering hurriedly through a familiar intersection. I sped past a green traffic light; a police car, sirens off, shot through a red light at the same time, knocking me cleanly off my scooter. I rolled onto the hood of the car, then slammed into the ground, inducing a rush of pain and blood. As I was loaded into an ambulance, the police officer took my backpack and driver's license to make his report, the first items I would be parted from that day. In the ambulance, the paramedic asked about medications and allergies, what parts of my body hurt, and if I had received my COVID vaccine. "Just questions I ask all my patients," she said benignly. I smiled wryly. That was a phrase I'd used many times.

"Trauma Central, Trauma Central, Trauma Central," my care team kept repeating. When an unidentified patient arrives at the hospital, they are given placeholder names in the rush to treat their acute injuries. Such placeholder names are the equivalent of the colloquial term "John Doe." My name had been replaced with the placeholder Trauma Central. I was quickly rushed through the emergency department on a stretcher and switched to the trauma bed, where my clothes were cut off to assess my medical status. "Twentyfour-year old male, suspected broken wrist, suspected ankle sprain, unidentified shoulder swelling, bleeding profusely above his eye." My medical team theorized about my injuries. Naked aside from a gown in the emergency department, this list of potential conditions was the only thing I had left to call my own.

I left the hospital that night in the standard post-trauma uniform: a nondescript white t-shirt with black shorts. The

Varun S. Mehta

varunmehta@gwu.edu

1 George Washington School of Medicine and the Health Sciences, Washington, DC, USA paper bracelet on my arm, my one identifier, marked my name as Trauma Central. As for my blood-stained backpack, identification card, and clothes, I had forgotten them at the hospital. The identity-effacing medical care assembly line. We boil our patients down to a list of conditions, and then hopefully build them back into complete humans after we've addressed their emergency. Trauma Central's final diagnoses were a left scaphoid waist fracture, right AC joint sprain, and left ankle sprain.

I returned to my psychiatry rotation just one week after the accident with a cast, sling, and ankle brace. This quick turnaround was of my own making. I was anxious to get back into the hospital, to be a provider rather than a patient.

The second patient I encountered that morning was hardly 5 years older than me. I scanned through his chart: 29-yearold male, psychosis, substance abuse disorder, history of trauma. Another patient stripped down to a list of diagnoses. In his patient room, I asked whether he ate, went to the bathroom, had thoughts of self-harm, and had been seeing things other people couldn't see. "Just questions I ask all my patients," I said benignly.

His hostility in response surprised me, his face a mask of irritation. "All you hospital people do is ask me the same questions every day! I'm a person, start treating me like one!". "I-uh-I'm sorry, I didn't mean to-." I stood fumbling for words, a classic sputtering medical student. I had intended to segue into a deeper understanding of his story, to make him feel like a person and not a Trauma Central. Instead, I'd been thrown off balance, accidentally creating the problem I had come to address.

Somehow, the patient himself came to my aid. "What's that on your wrist?," he asked perceptively. I sensed a subtle shift in his tone. Grateful to change the subject, I relayed my situation to him, explaining the cast. I showed him my ankle brace. He nodded, "I've been hit by a car before. Hope you feel better." I made cautious eye contact. The irritation was gone, his mask lifting to reveal a somber expression and a pair of sad brown eyes. I met them not with mine but with the eyes of Trauma Central. 
"I know my questions are repetitive. I know you barely feel like a human these days. But still, could you tell me your story?." I glanced at his paper wristband, realizing I had never addressed him by name. It didn't even matter. As if duty-bound to trust me with his story just as I had trusted him with mine, he explained all the events that led him to the hospital, a tale of a motor vehicle and shooting accident leading to job loss, substance use, and mental health difficulties. To anyone outside the room, a medical student went to interview a patient and reported back. But in that hospital room, the patient really spoke to Trauma Central, creating a bond of shared identity lost to the medical assembly line. The lines between patient and provider blurred, resulting in a beautiful display of understanding and humanity.

As my injuries healed in the following months, my identity as a recent hospital patient also began to visibly evaporate to my patients, and the barrier between hospital staff and patient re-formed in my clinical encounters. I'm not sure how to retain those brief humane connections I formed as Trauma Central. Maybe as I grow as a physician, I will find some answers, but I suspect they will not come easily.

\section{Declarations}

Disclosures The author states that there is no conflict of interest.

Publisher's Note Springer Nature remains neutral with regard to jurisdictional claims in published maps and institutional affiliations. 